\title{
Miradas sobre la calidad de la educación básica en Iberoamérica: Visiones de España y Colombia
}

\author{
Perspectives on the quality of basic education in Ibero-America: Spain and \\ Colombia insights
}

Pontos de vista sobre a qualidade da educação básica na Ibero-América:
visões da Espanha e da Colômbia

\author{
Alexander Javier Montes Miranda ${ }^{1}$ \\ Universidad de Cartagena, Colombia \\ Audin Aloiso Gamboa Suárez ${ }^{2}$ \\ Universidad Francisco de Paula Santander, Colombia
}

Recepción: 12/03/2018

Evaluación: 08/05/2018

Aceptación: 29/06/2018

Artículo de Investigación - Reflexión

DOI: $10.19053 / 01227238.8721$

\section{RESUMEN}

La educación básica es la encargada de consolidar las estructuras del sistema educativo. En gran parte del mundo los países han hecho esfuerzos importantes en materia de cobertura y en mayor medida aquellos orientados a mejorar su calidad. No obstante, cada vez más la calidad de la educación viene haciendo protagonista en los debates en este campo, desde los enfoques de desarrollo social y económico especialmente. De este modo, el presente artículo de investigación muestra la relación de dichas políticas en España y Colombia, como una forma de reconocer elementos comunes y divergentes. El texto hace parte del proyecto de una amplia investigación titulada políticas de calidad de la educación básica primaria en Co- lombia (1994 - 2015) y utiliza como horizonte metodológico el paradigma cualitativo. Los resultados revelan que la calidad de la educación es un concepto asociado a las metas de aprendizaje, que desde el modelo de competencias se traduce en lo que se espera deba saber y saber hacer un estudiante luego de su escolaridad, lo cual debe ser evaluado a nivel interno y externo.

Palabras clave: Revista historia de la educación latinoamericana; calidad de la educación; política educativa; educación básica.

\section{ABSTRACT}

Basic education is responsible for consolidating the structures of the education system. Many countries in the

1 Doctor en Ciencias de la Educación, RUDECOLOMBIA (Universidad de Cartagena). Miembro del grupo de Investigación Rueca. ORCID: http://orcid.org/0000-0002-7168-6295. Correo electrónico: amontes20@gmail.com.

2 Doctor en Ciencias de la Educación, RUDECOLOMBIA (Universidad de Cartagena). Docente investigador de la Universidad Francisco de Paula Santander (Cúcuta). ORCID orcid.org/0000-0001-9755-6408. Correo electrónico:audingamboa@ufps.edu.co. 
world have made significant efforts in terms of coverage and, to a greater extent, for the purpose of improving quality. However, more and more often the quality of education is the protagonist in this field debates, especially from social and economic development approaches.

This research article shows the relation of these policies, in Spain and Colombia, as a means to recognize common and divergent elements. This text is part of an extensive research work entitled "Quality policies of primary education in Colombia (1994 - 2015)" whose methodological framework is the qualitative paradigm. The results reveal that the quality of education is a concept associated with learning goals, and from the competency model is established what a student should know and know how to do after completing his schooling, this process must be evaluated internally and externally.

Palabras clave: Journal History of Latin American Education; Quality of education; education policy; basic education.

\section{RESUMO}

A educação básica é responsável pela consolidação das estruturas do sistema educacional. Em grande parte do mundo, os países fizeram esforços importantes em termos de cobertura e, em maior medida, os que visam melhorar sua qualidade. No entanto, cada vez mais a qualidade da educação tem desempenhado um papel de liderança nos debates neste campo, especialmente a partir de abordagens de desenvolvimento social e econômico. Dessa forma, o artigo de pesquisa apresentado mostra a relação dessas políticas na Espanha e na Colômbia, como forma de reconhecer elementos comuns e divergentes. $\mathrm{O}$ texto faz parte do projeto de uma extensa pesquisa intitulada políticas de qualidade da educação básica básica na Colômbia (1994 - 2015) e utiliza como horizonte metodológico o paradigma qualitativo. Os resultados mostram que a qualidade da educação é um conceito associado com os objectivos de aprendizagem, o que se traduz a partir do modelo de competências é esperado deve conhecer e saber como um estudante após a sua escolaridade, o que deve ser avaliado nível interno e externo.

Palavras-chave: Revista Historia da Educação Latino-americana; qualidade da educação; política educacional; Educação básica.

\section{INTRODUCCIÓN}

Los análisis referidos en el presente artículo son producto de la investigación políticas de calidad de la educación en básica primaria en Colombia (1994-2015), considerando que la calidad de la educación primaria en este país ha tenido fuertes cuestionamientos, especialmente por los resultados de las evaluaciones nacionales e internacionales, con posiciones marginales frente a otros países de Latinoamérica y del mundo ${ }^{3}$. A partir de lo cual, puede deducirse un problema educativo vertebral de todo el sistema, toda vez que este nivel es el encargado de consolidar las bases de los aprendizajes esperados en todo el proceso escolar de una persona, de manera que puede comprenderse, por ello, los bajos resultados

3 Alexander Montes, Zilath Romero y Audin Gamboa. "La formación docente en el marco de la política de calidad de la educación básica en Colombia». Revista Espacios vol. 38, no. 20 (2017). 
de la educación básica secundaria y media, así como también los que se tienen en los niveles superiores.

Una manera de evaluar el sistema educativo colombiano son los resultados de las pruebas PISA (Programa Internacional de Evaluación de Estudiantes), realizadas por la Organización para la Cooperación y Desarrollo Económico (OCDE), demostrando resultados deficientes en las áreas de matemáticas, lectura y ciencias ${ }^{4}$. Colombia inició la presentación de sus estudiantes a esta evaluación en el 2006, desde entonces no se han notado avances importantes en materia de resultados académicos de sus estudiantes, en ninguna de las áreas evaluadas, salvo en el año 2015, donde el resultado fue levemente positivo.

Para el caso de la educación básica, las pruebas SABER, realizadas por el instituto para la evaluación de la calidad de la educación (ICFES), también muestra un panorama preocupante. En las áreas evaluadas por las pruebas nacionales en ambos casos los resultados son mayoritariamente deficientes, para el caso del lenguaje más del $60 \%$ de los estudiantes evaluados se encuentran en niveles insuficientes y mínimos lo que quiere decir que la gran mayoría de estudiantes muestra un desempeño mínimo en las competencias exigibles para el área y el grado evaluado o en su defecto no demuestra los desempeños mínimos establecidos. La situación es mucho peor para el área de matemáticas donde los estudiantes colombianos en un porcentaje superior al 70\% demuestran niveles insuficientes en las competencias evaluadas.

Sin embargo, desde el año 1994, posteriormente a la definición de la actual Constitución Política de Colombia ha tratado de fortalecer el sistema educativo con normas, programas y procesos que se han enfocado en procesos organizativos, curriculares, evaluativos hasta llegar a la formación del profesorado, no obstante el panorama ha permanecido a lo largo de la historia, dado que, como se evidencia en los resultados generales que se acaban de describir, seguimos cosechando resultados preocupantes para los estudiantes de todos los niveles, especialmente de la básica primaria.

Para el caso de esta investigación, se analizan paralelamente los avances en política educativa de calidad de España y Colombia, como una manera de evaluar avances importantes en esta materia en nuestro país.

\section{Tensiones sobre el concepto de calidad de la educación}

En lo que se refiere al concepto de calidad de la educación, este análisis ha planteado dos tendencias teóricas, descritas a continuación:

El primero de ellos, está asociado a las dinámicas de mercado, en relación con la satisfacción del cliente, esta tendencia sostienen que los procesos de calidad adoptados en el sector industrial ${ }^{5}$ son perfectamente aplicables al educativo, por ello sobresalen indicadores de eficiencia, cobertura, análisis económicos de efec-

4 “Principales resultados PISA 2012". (Bogotá, 2012) Istituto Colombiano para el Fomento de la Educación Superior, (ICFES).

5 Audin Gamboa, Calidad de la educación superior: pretensiones y realidades institucionales. (Bogotá: Ecoe Ediciones, 2016$), 78$. 
tividad entre otros proceso relacionados con las dinámicas de oferta y demanda. Dentro de este modelo se encuentran los sistemas de acreditación de la calidad de las escuelas, muy comunes en Colombia y Latinoamérica ${ }^{6}$.

El segundo enfoque se denomina el triángulo de la tensión entre la autoridad Estatal, la oligarquía académica y las leyes del mercado ${ }^{7}$. Esta tendencia tiene un marcado interés en los análisis de costo resultado que hacen los gobiernos sobre las políticas educativas que proponen, aquí las instituciones son eficientes en la relación costo-resultados, propuesta evidente, en la década de los 80 con la preocupación de los estados por la contribución de la educación y de la formación en los resultados de las economías nacionales ${ }^{8}$. Finalmente, existe una marcada tendencia, desde una perspectiva mucho más crítica, ligada a lo educativo y que comprende la calidad en relación con los fines de la educación misma9.

\section{La política educativa como instrumento de poder}

De otro lado, dentro de los retos más importante para el análisis se consideró la comprensión de las pedagogías críticas como fundamento para la interpretación como posibilidad metodológica y pedagógica.

En este sentido, coinciden visiones del sentido de lo humano como condición primaria de la pedagogía ${ }^{10}$, lo que pone de manifiesto la necesidad de cualquier política educativa por comprender el contexto real en el que se desarrollarán y los actores involucrados en su implementación; vistos estos como seres humanos racionales y sociales mas no como máquinas o receptores pasivos de unas ideas centralizadas que no tienen la posibilidad de interlocución ${ }^{11}$.

Otro de los retos de las pedagogías críticas, tiene que ver con el reconocimiento, participación y visibilización de los grupos sociales minoritarios a los cuales se les ha desconocido e invisibilizado su propia voz y sentido ${ }^{12}$, ello lleva implícito un discurso de igualdad, justicia social y democracia, indispensables en la comprensión del fenómeno educativo en todos los niveles. Asimismo, la construcción de las identidades sociales, viene a ser un reto importante en este marco.

6 Nanci Barrios, “Calidad de las escuelas bolivarianas en Venezuela”. (Tesis Doctoral en, Universidad de León, 2011), 15.

7 Mario De Miguel Díaz, "La evaluación de los centros educativos. Una aproximación a un enfoque sistémico". Revista de Investigación Educativa, vol. 15, no. 17 (1997): 145-178.

8 Rosario Beresaluce, "La calidad como reto en las escuelas de educación infantil al inicio del siglo XXI: las escuelas de Reggio Emilia, de Loris Malaguzzi, como modelo a seguir en la práctica educativa”. (Tesis Doctoral en, Universidad de Alicante, 2008), 38

9 Alexander Montes, Audin Gamboa y Diana, Lago, "La educación básica en Colombia: Una mirada a las políticas educativas". Saber, Ciencia y Libertad, vol. 8, no. 2 (2013): 141-155; Diana Elvira Soto Arango; José Rivadeneira; Jorge Duarte; Sandra Liliana, Bernal Villate. "La generación del movimiento estudiantil en Colombia. 1910-19241", Revista Historia de la Educación Vol. 20 no. 30 (2018): 225.

10 Joel Kincheloe; La pedagogía crítica en el siglo XXI: evoluacionar para sobrevivir, pedagogía crítica - de qué hablamos, dónde estamos (Barcelona: Garó, 2008), 97.

11 Frank Blackler, "Knowledge, knowledge work and organizations: An overview and interpretation". Organization studies, Vol. 16, No. 6 (1995): 1021-1046.

12 Hugo Edgardo Bagiano. "La cultura de la resistencia juvenil y el proceso emancipador." Revista Historia de la Educación Latinoamericana, 11 (2008): 59-76. 
Por su parte, para algunos autores estas corrientes de pensamiento han influido instituciones económicas, políticas y culturales, a lo cual no escapa la escuela, generando una tensión constante entre las leyes del mercado y los valores tradicionales como condiciones determinantes de los campos cultural, social y educativo, estas tensiones plantean la discusión entre las posibilidades de privatización de las instituciones y las responsabilidades del estado en la garantía de los derechos de las personas, entre ellos el de la educación ${ }^{13}$.

A lo anterior, se le suma la visible propuesta del neoliberalismo por alinear el sentido de la escuela con el mundo laboral, estamos hablando de una escuela que forme para los intereses de la empresa y para las demandas de la economía, a lo que se le ha demonizado proceso Mcdonalización. Ello también ha llevado a que el discurso de la educación y de la escuela cada vez se homologue más a esta dinámica ${ }^{14}$. Sin embargo, es evidente que este tipo de visiones aíslan la garantía de una educación de calidad con equidad.

\section{Metodología}

El presenta trabajo empleó el enfoque teórico de la investigación cualitativa. Dado que se sustenta en una visión teórica interdisciplinaria, mediante el empleo de la metodología del análisis de contenido. Empleando la ruta con el siguiente ciclo:

Esquema Teórico: se refiere a la construcción teórica que se utiliza como referencia para los análisis. Tipo de muestra: esta segunda fase consiste en la selección de los documentos y personas que serán la fuente de los datos para el análisis. Sistema de códigos o categorías: este proceso se construye luego de la revisión teórica y se realiza con el fin de poder sintetizar el análisis en aspectos claves para el cumplimiento de los objetivos de la investigación y el control de calidad que se refiere al proceso final de validación del análisis documental ${ }^{15}$.

La definición de las categorías y los códigos son formas de organizar el raciocinio para el análisis del objeto de estudio, en esta oportunidad, tal como lo plantea el autor ayudan en la configuración de la ruta de análisis documental propuesto en esta investigación ${ }^{16}$.

Para la selección de los documentos en función de los objetivos de investigación y de las categorías definidas se consideraron los documentos oficiales de política de calidad educativa propuestos desde el Ministerio de Educación en

13 Enrique Javier Díaz. "La globalización neoliberal y sus repercusiones en educación". Revista electrónica interuniversitaria de formación del profesorado, Vol. 13, No. 2 (2010): 23-38.

14 Diana Elvira Soto. "Legislación e imaginarios sociales en el escalafón y los salarios de los educadores de primaria en Colombia. 1952-19941". Revista Historia de la Educación Latinoamericana, Vol. 15, No. 21 (2014): 229-262. Doi. https://doi. org/10.19053/01227238.2472.

15 Abela Andreú, Las técnicas de análisis de contenido: una revisión actualizada. (Documento en línea, 1998), http://public. centrodeestudiosandaluces. es/pdfs S 200103.1 (2 de agosto, 2018).

16 Maria Ciavatta. "Quando nós somos o outro: questões teórico-metodológicas sobre os estudos comparados. Educação \& Sociedade,vol. 21 , no. 72 (2000): 65-79. 
Colombia y los documentos de legislación Español, en el período de tiempo determinado en la investigación, con base en los criterios de suficiencia y cantidad.

\section{Resultados}

Visión de la Calidad de la educación en el desarrollo de la política educativa. Caso de España

En el siguiente apartado se describirá la legislación española en materia de ordenamiento y política de calidad de la educación básica desde 1990 hasta la actualidad, analizando, de este modo, las cuatro leyes orgánicas que han existido en este periodo (1/1990, 10/2002, 2/2006 y 8/2013), haciendo especial énfasis en la actual ${ }^{17}$, toda vez que centra su atención de manera puntual en la calidad de la educación de este país. Ello, con el propósito de conocer un punto de referencia de la manera como se ha concebido este nivel educativo en otros países.

En primer lugar, la Ley Orgánica 1/1990, de 3 de octubre, de Ordenación General del Sistema Educativo - LOGSE -, que derogó la Ley Orgánica del Estatuto de Centros Escolares, se dedicó al ordenamiento del sistema educativo español, determina la aplicación y seguimiento de la ley en el marco de la enseñanza pública, privada y privada concertada, esta última modalidad se refiere a los particulares que contratan por el servicio educativo, figura conocida en Colombia como banco de oferentes. Esta ley se ampara en los mandatos constitucionales que garantizan las libertades de enseñanza, de didáctica y de creación de centros, así como el derecho a recibir formación religiosa y moral de acuerdo con las propias convicciones. Aspecto relevante en la coherencia legislativa y política de los estados, toda vez que el desarrollo de los sistemas educativos requiere especial alineación con los derechos fundamentales de las personas expresados en sus cartas constitucionales.

Vale la pena aclarar, que esta ley tiene como antecedente el reconocimiento de los logros en materia educativa que ha tenido España en las décadas anteriores traducidas en los alcances de la escolarización total en la educación general básica, creándose para ello un gran número de puestos escolares y mejorando las condiciones de otros ya existentes, se ha incrementado notablemente la escolarización en todos los niveles no obligatorios, se han producido importantes avances en la igualdad de oportunidades, tanto mediante el aumento de becas y ayudas como creando centros y puestos escolares en zonas anteriormente carentes de ellos, se han producido diversas adaptaciones de los contenidos y de las materias. Las condiciones profesionales en que ejerce su función el profesorado difieren, cualitativamente, de las entonces imperantes ${ }^{18}$.

Uno de los aspectos más relevantes planteados en esta ley es la vinculación de los padres, profesores, alumnos y la sociedad en general en las responsabilidades del sistema educativo, de manera que asigna derechos y deberes vitales

17 "Ley Orgánica para mejorar la calidad educativa" (España, 2013), Ordenación General del Sistema Educativo (LOGSE).

18 "Ley Orgánica para mejorar la calidad educativa” (España, 1990), LOGSE. 
para el cumplimiento de los fines de la educación expresados en la misma ley, así mismo, se establece en esta normativa la condición de gratuidad y obligatoriedad de la educación Básica, características vigentes en la normativa colombiana pero difíciles de conquistar hasta los tiempos actuales.

Esta ley, propone también, una reordenación del sistema educativo en sus otros niveles estableciendo en su régimen general las etapas de educación la educación primaria, educación secundaria, que comprenderá la educación secundaria obligatoria, el bachillerato y la formación profesional de grado medio-, la formación profesional de grado superior y la educación universitaria (Ley Orgánica 1/1990). En coherencia con ello, el periodo común, entendido en la concepción de la educación básica obligatoria y garantizada por el estado incluye diez años de escolaridad, en ella se integra la etapa de la educación primaria y la secundaria.

De igua manera, la Ley Orgánica 10/2002 ${ }^{19}$, de 23 de diciembre plantea de manera puntual una reforma que solucione los índices de abandono escolar que estaban, en ese momento, en el $25 \%$, promedio que superior a la media de la Unión Europea, de manera, que abiertamente el concepto de calidad se asocia en esta reforma al problema de la cobertura y la permanencia. Asimismo se proponen como fines de esta reforma el mejoramiento de los niveles de formación de los estudiantes, impulsar sistema efectivo de oportunidades para todos, fomentar la cultura del esfuerzo y de la evaluación y mejorar las condiciones para el desarrollo de la función docente.

Dentro de los aspectos más relevantes de esta reforma se puede encontrar la gratuidad de la educación infantil; la supresión de la promoción automática (asunto resuelto en Colombia en el año 2009 con el decreto 1290); la implantación de itinerarios para satisfacer las necesidades y expectativas de los alumnos; el establecimiento de una prueba general de bachillerato, como ocurre en la mayor parte de los países de la UE, para homologar los conocimientos de los alumnos; la creación de programas de iniciación profesional; el establecimiento de una verdadera carrera docente con mayores posibilidades de promoción, dando más importancia a su formación, tanto inicial como permanente, y con medidas de apoyo; el refuerzo de las competencias de los directores y de la autonomía de los centros; la adopción de medidas para mantener la convivencia en las aulas; y el refuerzo de la lectura y de las materias instrumentales en todos los niveles educativos.

Frente al proceso de evaluación de los aprendizajes se propone un modelo continuo, sin embargo, como quiera que se elimina la promoción automática, un estudiante podría repetir, solo por una vez un año escolar, cuando no alcance los objetivos propuestos en el currículo; sin embargo, cuando un estudiante sea promovido con deficiencias deben garantizárseles apoyos necesarios para que pueda recuperar dichas deficiencias.

19 "Ley Orgánica para mejorar la calidad educativa" (España, 2002), LOGSE. 
Seguidamente, la LOGSE plantea los fines de la educación primaria, esta tiene como objetivo profundizar en los procesos lectoescriturales y de cálculo, al punto que todas las áreas del currículo deberán incluir actividades que fomenten la lectura, de manera que el fin principal de este nivel radica en el desarrollo de habilidades en estos campos, lo que obliga un rediseño del currículo para la enseñanza en este nivel potenciando las áreas instrumentales (Lengua y Matemáticas) y las Lenguas Extranjeras (se adelantará el aprendizaje de una lengua extranjera al primer curso de primaria, que hoy comienza en tercero

Finalmente, es importante resaltar la concepción de calidad de la educación planteada en el capítulo uno de esta reforma, donde se reafirman los principios desde los cuales se comprende este índice rector de la ley, enunciados según los siguientes criterios: la equidad, como principio de garantizar educación para todos; la capacidad de transmitir valores que favorézcan la libertad personal, la responsabilidad social, la cohesión y mejora de las sociedades y la igualdad de derechos entre sexos que ayuden a superar cualquier tipo de discriminación.

Otra reforma al sistema educativo español fue planteada en la Ley Orgánica 2/2006, con el propósito fundante de garantizar una escolarización más prolongada y con unas metas más ambiciosas, considerando que la universalización de la enseñanza primaria ya era una realidad desde finales del siglo XIX. Además de ello, esta reforma se fundamenta, como lo habían expresado las anteriores en la búsqueda de unas condiciones de alta calidad en condiciones de equidad.

Esta ley, es el producto de compromisos españoles frente a comunidades internacionales tales como el acuerdo firmado en parís en 1990, donde asistieron los ministros de educación de los países de la OCDE, cuyo fin era la búsqueda de alternativas que garantizaran una educación y una formación de calidad para todos, apuesta a la cual se sumaron los países de mayor desarrollo. De la misma manera, este compromiso fue adquirido en la 47.. Conferencia Internacional de Educación convocada por la UNESCO en Ginebra, donde participaron más de sesenta ministros.

Así las cosas, esta ley se plantea desde tres principios fundamentales, a saber: La exigencia de proporcionar una educación de calidad a todos los ciudadanos de ambos sexos, en todos los niveles del sistema educativo; la necesidad de que todos los componentes de la comunidad educativa colaboren para conseguir ese objetivo tan ambicioso. La combinación de calidad y equidad que implica el principio anterior exige ineludiblemente la realización de un esfuerzo compartido y el compromiso decidido con los objetivos educativos planteados por la Unión Europea para los próximos años.

De esta manera, puede notarse que las motivaciones más importantes para reformar la política educativa en España, tiene que ver con metas establecidas en el marco de su participación en comunidades internacionales, que veían en la educación un elemento importante de competitividad y desarrollo, en la medida que se trabajaran de manera conjunta en el cumplimiento de principios de calidad. 
Para el cumplimiento de estos fines de calidad se propusieron desde la política estrategias que combinaran el proceso de autonomía pedagógica y de gestión de los centros docentes con mecanismos de evaluación y de rendición de cuentas. De manera que este proceso de rendición de cuentas buscaba el desarrollo de procedimientos de evaluación que involucrara diferentes ámbitos y agentes del sistema educativo (alumnado, profesorado, centros, currículo, administraciones) articulado con un sistema de rendición de cuentas de los avances en la gestión de los mismos centros.

Por último, se presentan los planteamientos en esta materia hechos en la Ley Orgánica 8/2013, de 9 de diciembre, para la mejora de la calidad educativa. Dicha reforma fue planteada con el objetivo expreso de ubicar la educación en el centro de la sociedad y la economía, de manera que de entrada se conjugan dos contextos que han sido objeto de debate en los estudios educativos, las dinámicas propias del mercado y la escuela, que, sin embargo, aquí aparecen como puntos de convergencia.

Son tres las principales razones que motivaron esta reforma: los resultados obtenidos por los alumnos y alumnas en las pruebas de evaluación internacionales como PISA, las elevadas tasas de abandono temprano de la educación y la formación, las cuales se encontraban en el $26,5 \%$ en 2011, con tendencia al descenso pero muy lejos del valor medio europeo actual (13,5\%) y del objetivo del 10\% fijado para 2020. Y por último el reducido número de estudiantes que alcanza la excelencia. Así mismo, esta apuesta se debe a recomendaciones de la OCDE basadas en las mejores prácticas de los países con sistemas educativos con mejores resultados, en los que las reformas se plantean de manera constante sobre un marco de estabilidad general según se van detectando insuficiencias o surgen nuevas necesidades.

Con base en todo lo anterior, la ley orgánica actual de calidad de la educación en España plantea los siguientes aspectos generales para la búsqueda de la calidad de la educación en este país: el aumento de la autonomía de centros, el refuerzo de la capacidad de gestión de la dirección de los centros, las evaluaciones externas de fin de etapa, la racionalización de la oferta educativa y la flexibilización de las trayectorias.

El primero de estos factores, autonomía de los centros, hace parte de una recomendación expresa de la OCDE que tiene como fin el mejoramiento de los mismos, sin embargo dicha autonomía se plantea asociada con un proceso de rendición de cuentas, con ello se busca dar cuenta de la eficiencia de la inversión de los fondos públicos. Es necesario que cada centro tenga la capacidad de identificar cuáles son sus fortalezas y las necesidades de su entorno, para así poder tomar decisiones sobre cómo mejorar su oferta educativa y metodológica en ese ámbito.

En lo que tiene que ver con las evaluaciones externas, de carácter formativo y diagnóstico, encargadas a las pruebas PISA, garantizarán la homogenización de la titulación en toda España y sirven como indicador para la evaluación de la gestión directiva de las escuelas, dichas evaluaciones son aplicadas con el pro- 
pósito que los estudiantes alcancen los niveles de aprendizaje adecuados para el normal desenvolvimiento de la vida personal y profesional conforme el título pretendido, y además deben permitir orientar a los alumnos y alumnas en sus decisiones escolares de acuerdo con los conocimientos y competencias que realmente posean como lo señala la Ley Orgánica 8/2013.

Puntualmente, para el caso de la educación primaria, se concibe en esta ley la evaluación final de la primaria realizado al finalizar el grado sexto, aplicada individualmente a cada alumno en la que se comprobará el grado de adquisición de la competencia en comunicación lingüística, de la competencia matemática y de las competencias básicas en ciencia y tecnología, así como el logro de los objetivos de la etapa, de los resultados de esta evaluación se presentará informe detallado a los padres de familia, proponiendo planes de mejorar aquellos estudiantes que presenten dificultades.

\section{Desarrollo de la política educativa en materia de organización del sistema educativo}

Es importante resaltar que el presente análisis se realizó a partir de los lineamientos generados en la Ley General de Educación ${ }^{20}$

Para contextualizar, es necesario precisar que en el año 1991 la Asamblea Nacional Constituyente decretó la Constitución Política de Colombia, en el artículo 67 se propone la educación como un derecho y un servicio público que tiene una función social, en este momento los fines del sistema se centran en el acceso al conocimiento, a la ciencia, a la técnica, y a los demás bienes y valores de la cultura, también es su función la formación en el respeto a los derechos humanos, a la paz y a la democracia; y en la práctica del trabajo y la recreación, para el mejoramiento cultural, científico, tecnológico y para la protección del ambiente.

Este concepto de obligatoriedad pone en manos del estado una responsabilidad que históricamente había estado asumido en gran parte por la iglesia, y poder resaltar las falencias en posibilidades de acceso al mismo sistema. De modo que el problema de cobertura se ponía en el centro de la preocupación del estado y su política. No obstante, el control, la supervisión de la calidad del mismo sistema y el cumplimiento de los fines era un tema del control y ejercicio exclusivo del estado.

Así, tiene el estado la responsabilidad constitucional de garantizar la calidad y el cumplimiento de los fines de la educación, mandato base para la definición de una Ley General de Educación que fue aprobada el 8 de octubre de 1994, coherente con estos principios constitucionales.

La Ley General de Educación en su Artículo 1 define el concepto de la educación como proceso de formación permanente personal, cultural y social que se fundamenta en una concepción integral de la persona humana, de su dignidad, de sus derechos y de sus deberes.

20 “Ley General de Educación 115" (Bogotá: 1994), MEN. 
El artículo cuatro de esta Ley define los principios de calidad y cubrimiento del servicio, recalcando la responsabilidad del estado de velar por la calidad de este servicio, que le obliga a ejercer suprema inspección y vigilancia mediante las instituciones creadas para tal fin. El Estado deberá atender en forma permanente los factores que favorecen la calidad y el mejoramiento de la educación; especialmente velará por la cualificación y formación de los educadores, la promoción docente, los recursos y métodos educativos ${ }^{21}$, la innovación e investigación educativa, la orientación educativa y profesional, la inspección y evaluación del proceso educativo.

Es así como se plantean los tres niveles de la educación formal: el preescolar que comprenderá mínimo un grado obligatorio, la educación básica con una duración de nueve (9) grados que se desarrollarán en dos ciclos: la educación básica primaria de cinco (5) grados y la educación básica secundaria de cuatro (4) grados y la educación media con una duración de dos (2) grados.

En términos de calidad, esta ley inicia la construcción de un plan normativo que defina las acciones, fines y procesos necesarios para dar cumplimiento a los principios constitucionales en esta materia; de este modo, la primera política construida en el marco de esta ley es el Plan Nacional de Desarrollo Educativo. Este plan tiene carácter de decenal, el primero de ellos debía ser construido en dos años, a partir de la promulgación de la presente ley, cubrirá el período de 1996 a 2005 e incluirá lo pertinente para que se cumplan los requisitos de calidad y cobertura.

En términos curriculares, esta ley también define los principios generales, inicia por la concepción del Proyecto Educativo Institucional (PEI), en el cual cada establecimiento educativo debe materializar los fines, aspectos generales y proyectos mediante los cuales garantizarán educación de calidad en todos los niveles que ofrece, además de los recursos docentes didácticos disponibles y necesarios, la estrategia pedagógica, el reglamento para docentes y estudiantes y el sistema de gestión, este proyecto, debe responder a las necesidades de los educandos y de las características de los contextos específicos de formación, además de ser factible y evaluable como se sugiere en el Artículo 73 de la Ley 115 de 1994.

De este modo, se materializa en la política reciente colombiana, la idea de currículo, puntualmente en la etapa inicial de la educación, entendido en esta ley como el conjunto de criterios, planes de estudios, programas, metodología, y procesos que contribuyen a la formación integral y a la construcción de la identidad cultural nacional, regional y local, incluyendo también los recursos humanos, académicos y físicos para poner en práctica las políticas y llevar a cabo el Proyecto Educativo Institucional.

Dicha conceptualización curricular, es desarrollada en el decreto 1860 de 1994, el decreto 1290 de 2009, los lineamientos curriculares de las diferentes áreas y los

21 Armando Martínez Moya, "Libertad de cátedra o socialismo de Estado. El dilema de los estudiantes de la Universidad de Guadalajara en 1933-1937”. Revista Historia de la Educación Latinoamericana, vol. 16, no. 22 (2014): 191-211. DOI: http://dx.doi.org/ 10.9757/ Rhela.22/09 
estándares básicos de competencias, a lo cual se dedicará un capítulo especial en el presente estudio. Sin embargo, la misma ley brinda autonomía escolar en materia curricular a los Establecimientos educativos para organizar las áreas fundamentales de conocimiento definidas para cada nivel, introducir asignaturas optativas dentro de las áreas establecidas en la Ley, adoptar algunas áreas a las necesidades y características regionales, adoptar métodos de enseñanza y organizar actividades formativas, culturales y deportivas, dentro de los lineamientos que establezca el Ministerio de Educación Nacional, y le asigna la responsabilidad a las Secretarías de Educación Municipales y departamentales (Certificadas) en la asesoría para el diseño del mismo currículo.

\section{De la concepción del currículo a la estandarización de competencias}

En 1994, año en que se expidió la Ley 115, también se conoció el decreto 1860, encargado de desarrollar las nociones de currículos planteadas en la ley general de educación en los apartes referenciados anteriormente; de esta manera se presentan los criterios para la elaboración del currículo, entendido como el producto de un conjunto de actividades organizadas y conducentes a la definición y actualización de los criterios, planes de estudio, programas, metodologías y procesos que contribuyan a la formación integral y a la identidad cultural nacional en los establecimientos educativos.

Esta norma establece las definición de los artículos 77 y 78 de la Ley 115, dada la necesidad de los establecimientos educativos de mantener actividades de desarrollo curricular que permitan el diseño y la evaluación del currículo, en el marco de la autonomía que les confiera la ley; y, que además, estas respondan a las necesidades y características propias del medio sociocultural donde se apliquen.

No obstante, la autonomía señalada, el diseño curricular debe integrar las consideraciones de este decreto: los fines de la educación y los objetivos de cada nivel y ciclo definidos por la Ley 115 de 1994, los indicadores de logro que defina el $\mathrm{MEN}^{22}$ y los lineamientos que expida el Ministerio de Educación Nacional para el diseño de las estructuras curriculares, los procedimientos para su conformación; y la organización de las diferentes áreas que se ofrezcan ${ }^{23}$.

Para la comprensión de estos principios curriculares se iniciará por las definiciones específicas en materia de planes de estudio, en coherencia con la Ley 115 desarrollados en el decreto 1860, entendido como el conjunto de áreas, asignaturas y los proyectos pedagógicos que integran el PEI, los cuales deben contener al menos los siguientes aspectos: la identificación de los contenidos, temas y problemas de cada asignatura y proyecto pedagógico, así como el señalamiento de las diferentes actividades pedagógicas; la distribución del tiempo y las secuencias del proceso educativo, señalando el período lectivo y el grado en que se

22 "Resolución 2343" (Colombia, 1996), MEN.

23 "Artículo 33 del Decreto 1860" (Colombia, 1994), MEN. 
ejecutarán las diferentes actividades; la metodología aplicable a cada una de las asignaturas y proyectos pedagógicos, señalando el uso del material didáctico, de textos escolares, laboratorios, ayudas audiovisuales, la informática educativa o cualquier otro medio o soporte de la acción pedagógica; los logros para cada grado, o conjunto de grados, según los indicadores definidos en el PEI y los criterios de evaluación y administración del plan como lo contempla el artículo. 38 del Decreto 1860 de 1994.

Hasta este momento, la política educativa había integrado diferentes conceptos respecto al diseño curricular. En primer lugar, la identificación de los contenidos, temas y problemas de cada asignatura y proyecto pedagógico haciendo un énfasis especial en el desarrollo de unidades temáticas, lo cual cambió en 1996 con la Resolución 2343 de 1996, donde se plantearon los indicadores de logro por conjunto de grados, que hacía visible una nueva política en materia del modelo de formación por competencias, decisivo en las políticas actuales en el campo curricular.

Sin embargo, en el año 2002 el decreto 0230, derogado por el decreto 1290 de 2009, propone una nueva estructura del plan de estudios, manteniendo el concepto planteado en las normatividad previa; en esta nueva estructura el plan de estudios estaría integrado por: la intención e identificación de los contenidos, temas y problemas de cada área, señalando las correspondientes actividades pedagógicas; la distribución del tiempo y las secuencias del proceso educativo, señalando en qué grado y período lectivo se ejecutarán las diferentes actividades, los logros, competencias y conocimientos que los educandos deben alcanzar y adquirir al finalizar cada uno de los períodos del año escolar, en cada área y grado, según hayan sido definidos en el Proyecto Educativo Institucional, PEI, en el marco de las normas técnicas curriculares que expida el MEN.

¿Igualmente, incluirá los criterios y procedimientos para evaluar el aprendizaje, el rendimiento y el desarrollo de capacidades de los educandos; el diseño general de planes especiales de apoyo para estudiantes con dificultades en su proceso de aprendizaje; la metodología aplicable a cada una de las áreas, señalando el uso del material didáctico, textos escolares, laboratorios, ayudas audiovisuales, informática educativa o cualquier otro medio que oriente o soporte la acción pedagógica; indicadores de desempeño y metas de calidad que permitan llevar a cabo la autoevaluación institucional según lo señalado en el Artículo 3 del Decreto 230 de 2002.

Como consecuencia de las normas ya señaladas en el 2006, Colombia conoce la materialización de la estandarización de competencias, orientada a construir desde el año 2002, una política curricular que tenía como meta aportar en la consolidación de la calidad educativa en este País alcanzar una educación de calidad para todas y todos como condición para el desarrollo de las naciones ${ }^{24}$. De manera que el primer propósito de esta política es la calidad de la educación, al respecto, oficialmente se reconoce la complejidad del concepto, a razón de

24 "Estándares básicos de competencias" (Colombia, 2006), MEN. 
la naturaleza de la labor educativa, la diversidad de actores involucrados y la multiplicidad de factores que la afectan.

A pesar de la ambigüedad en la definición del concepto, se establece como consenso de la política la definición de unas metas en función de una situación deseada expresada en términos de lo que se espera que el estudiante logre en su paso por la escuela. Se trata de un concepto de calidad asociado con unas metas de aprendizaje, que desde el modelo de competencias se traduce en lo que se espera deba saber y saber hacer un estudiante luego de su escolaridad, lo cual debe ser evaluado a nivel interno y externo, de manera que entra a tener visibilidad otro de los factores asociados desde esta visión, como lo es la evaluación, ahora no solo en el aula sino de un sistema que a nivel externo de cuenta de los aprendizajes reales de los estudiantes.

\section{CONCLUSIÓN}

El sistema educativo, define condiciones que propicien la inclusión de todas las personas al sistema educativo, no obstante existen muchas dificultades en las escuelas para la implementación de este principio que no solo vincula a estudiantes con necesidades educativas especiales sino también a poblaciones minoritarias, grupos étnicos y cualquier población que constituya posibilidades de diferencia.

La evaluación se asocia a las posibilidades de mejoramiento tanto de la institución como de los estudiantes, así como de generar insumos para la valoración de los aprendizajes hasta determinar la promoción de los estudiantes; no obstante, la misma política hacía prácticamente automática la promoción de los estudiantes independiente de los resultados de dicha evaluación.

Se consideran importantes los aportes realizados por la Ley 115 de 1994 a la educación Colombiana, en materia curricular, de organización del sistema, autonomía y participación, no obstante, la definición del perfil docente ha afectado la calidad de la educación.

Frente a la Evaluación, los maestros reconocen una fortaleza en esta política en el enfoque Formativo e integral de la misma, además de la participación de todos los actores en el proceso, no obstante en el Decreto 230 de 2002, se evidencia un aporte negativo al desarrollo educativo del país por la determinación de la promoción prácticamente automática de los estudiantes, ya que ello significó bajo rendimiento académico, motivación y responsabilidad de parte del estudiante. Asunto que fue resuelto por el decreto 1290 de 2009, que otorgó la autonomía de la definición de los criterios de la evaluación y promoción a la escuela, no obstante el MEN ha seguido, por otros medios teniendo injerencia en la promoción de los estudiantes.

El desarrollo de la política educativa colombiana en el ciclo básico puede resumirse en un proceso cíclico que integra tres componentes, a saber: La evaluación, el currículo estandarizado y las prácticas pedagógicas. 
La estandarización es una respuesta al campo de poder económico externo de la escuela, y su interés por universalizar los aprendizajes de los estudiantes, alineado con las tensiones ideológicas entre el neoliberalismo y neoconservadurismo. Y por tanto responde a una necesidad de medición, que viene a ser principio de la concepción de la calidad implícita en la política educativa colombiana

El desarrollo de la política educativa en materia de evaluación separa dos enfoques principales de la evaluación. La interna realizada por la escuela y la externa, realizada por el estado, mediante su organismo evaluador (ICFES).

La evaluación externa, ha tenido como propósito ser el instrumento del indicador de calidad primario evidente en la misma política, como son los resultados de dichas evaluaciones. Por esta razón, ha adquirido mayor desarrollo, especialmente con la implementación de la estandarización del currículo.

\section{FUENTES}

Instituto Colombiano para el Fomento de la Educación Superior (ICFES). Bogotá - Colombia. Principales resultados PISA 2012

Ley Orgánica para mejorar la calidad educativa (LOGSE), Madrid - España. Ordenación General del Sistema Educativo.

Ministerio de Educación Nacional (MEN), Bogotá - Colombia. Artículo 33 del Decreto 1860

Ministerio de Educación Nacional (MEN), Bogotá - Colombia. Resolución 2343.

Ministerio de Educación Nacional (MEN), Bogotá - Colombia. Estándares básicos de competencias

\section{REFERENCIAS}

Martínez, Moya Armando. “Libertad de cátedra o socialismo de Estado. El dilema de los estudiantes de la Universidad de Guadalajara en 1933-1937". Revista Historia de la Educación Latinoamericana vol. 16, no. 22 (2014): 191-211.

Montes, Alexander; Romero, Zilath y Gamboa, Audin. "La formación docente en el marco de la política de calidad de la educación básica en Colombia". Revista Espacios 38, no. 20: (2017).

Montes, Alexander; Romero, Audin; Lago, Diana. "La educación básica en Colombia: Una mirada a las políticas educativas". Saber, Ciencia y Libertad 8, no. 2 (2013): 141-155.

Romero, Audin, Calidad de la educación superior: pretensiones y realidades institucionales. Bogotá: Ecoe Ediciones, 2016.

Andreú, Abela. Las técnicas de análisis de contenido: una revisión actualizada. (Documento en línea, 1998), http://public. centrodeestudiosandaluces. es/pdfs S 200103.1 (2/08/2018).

Soto Arango, Diana Elvira. “Legislación e imaginarios sociales en el escalafón y los salarios de los educadores de primaria en Colombia. 1952-19941". Revista Historia de la Educación uLatinoamericana 15, no. 21 (2014): 229-262.

Soto, Diana; Rivadeneira, José; Duarte, Jorge; Bernal Villate, Sandra Liliana. “La generación del movimiento estudiantil en Colombia. 1910-19241", Revista Historia de la Educación Vol. 20 no. 30 (2018): 225.

Díez, Enrique Javier. “La globalización neoliberal y sus repercusiones en educación”. Revista electrónica interuniversitaria de formación del profesorado 13, no. 2 (2010): 23-38.

Blackler, Frank. "Knowledge, knowledge work and organizations: An overview and interpretation". Organization studies 16, no. 6 (1995): 1021-1046.

Bagiano, Hugo Edgardo. "La cultura de la resistencia juvenil y el proceso emancipador". Revista Historia de la Educación Latinoamericana 11 (2008): 59-76. 
Kincheloe, Joel. La pedagogía crítica en el siglo XXI: evolucionar para sobrevivir, pedagogía crítica - de qué hablamos, dónde estamos. Barcelona: Garó, 2008.

Díaz, Mario De Miguel. “La evaluación de los centros educativos. Una aproximación a un enfoque sistémico". Revista de Investigación Educativa 15, No. 17 (1997): 145-178.

Ciavatta, María. "Quando nós somos o outro: questões teórico-metodológicas sobre os estudos comparados". Educação \& Sociedade 21, no. 72 (2000): 65-79.

Barrios, Nanci. "Calidad de las escuelas bolivarianas en Venezuela". Tesis Doctoral en, Universidad de León, 2011.

Beresaluce, Rosario. "La calidad como reto en las escuelas de educación infantil al inicio del siglo XXI: las escuelas de Reggio Emilia, de Loris Malaguzzi, como modelo a seguir en la práctica educativa". Tesis Doctoral en Universidad de Alicante, 2008, 38.

Cómo citar:

Montes Miranda, Alexander Javier; Gamboa Suárez, Audin

Aloiso. “Miradas sobre la calidad de la educación básica en Iberoamérica: Visiones de España y Colombia" Revista Historia de la Educación Latinoamericana. Vol. 20 No. 31 (2018): 229-244 DOI: $10.19053 / 01227238.8721$ 